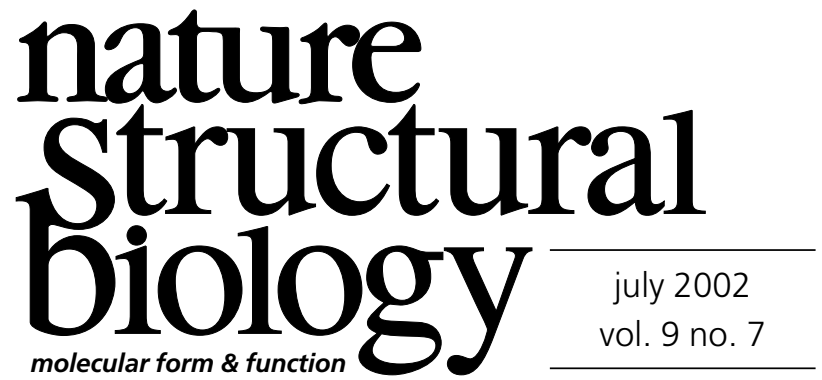

\title{
What is the point of a database?
}

This may seem a trivial question. Central repositories of Gigabytes of related information have become an essential part of every scientist's research. Therefore, questioning their utility is a bit like asking why we need oxygen. But as databases increase, both in size and number, and to ensure that they are as helpful as possible, we need to consider exactly what they are being used for.

A database is a tool for finding out what information is already known, and the quality of a database relies on three things: completeness, accuracy and accessibility. A database must be comprehensive and up-to-date; its content needs to be reliable or at least with well-documented accuracy. Finally, to be really useful a database should be simple to use - whoever, or whatever, comes to it for information must get what they want in a form they can understand.

The desire to provide comprehensive information is driving a proliferation of databases. To be truly comprehensive is an impossible task, so databases strive to be comprehensive within certain well-proscribed limits, but then every different set of limits spawns a new database. For example, practically every genome initiative has its own separate repository for all the sequences from its particular organism.

The limits can also be set by data that are useful to a particular community. A case in point is the Alliance for Cell Signaling (AfCS), the avowed aim of which is to map and document the entire web of interactions formed by a cell's signaling pathways. This is a very tall order, so rather than attempting to curate information for all cells at once, the collection is initially limited to two clinically relevant cell types: mouse lymphocytes and cardiomyocytes. In conjunction, the AfCS is developing a database of 'Molecule Pages', containing available information on molecules involved in mammalian cell signaling.

In many ways these Molecule Pages will form more of an encyclopedia than a conventional database; therefore, guaranteeing the accuracy of their content becomes critically important. The AfCS molecule pages will be updated regularly, authored by leading researchers and subjected to a form of peer review. Producing these pages thus constitutes a form of publishing. Indeed Nature is collaborating with the AfCS to help reviewing and distributing these resources.

In structural biology we have one of the oldest established of all biological databases, the Protein Data Bank. Incredibly it was effectively established in 1971 when only a handful of atomic resolution crystal structures had been solved. What catalyzed its formation were advances in computer graphics, which, despite having no more than $32 \mathrm{~K}$ of CPU memory to work with, allowed molecules of the size of proteins to be displayed relatively easily. For the graphic programs to work effectively across the entire database, the files needed to be in a standard format, including such basic concerns as whether the coordinates were measured in centimeters or inches. With the establishment of the PDB it was possible to look at any of the known protein structures in the same way.

From its inception the PDB has come a long way - quite literally, having moved from a centralized location at the Brookhaven National Laboratory to a consortium encompassing three sites, one as far as the San Diego Supercomputing Center. More importantly, the content has increased from a few structures to well in excess of 15,000 today, and is on course to have $\sim 45,000$ entries by 2005 . The completeness of the PDB has been assured by the policy of journals, including Nature Structural Biology, to demand that structures cannot be published in their pages without simultaneous deposition. The accuracy of the database is policed by suites of checking programs that attempt to detect errors in coordinates when they are submitted.

The shear volume of information now stored in the PDB puts an added premium on the consistency of data format, a situation that holds for all databases. Increasingly databases are interrogated, not by human users but automatically by bioinformatics programs, to identify ever more subtle patterns and connections in the available data. Consistent data format applies to both the primary experimental data and other associated information. In the case of the PDB, raw coordinates as well as the annotated information of a structure will need to be described in common and established ontology.

Ultimately there is much to be gained by establishing if not consistent, then at least compatible formats and ontology across a range of databases. With this compatibility, pieces of software called 'intelligent agents' will be able to extract related information from any and all databases in which it may reside. Such standardization would almost abolish the need for databases to be comprehensive because data would be available from more than one source. At the same time, comparing information gleaned from several sources could provide an assessment of the accuracy of those data. Consider this: by standardizing the way atomic coordinates are documented, the PDB has proven an invaluable boon to structural biology. How much more useful to bioinformatics, in all its guises, would be standardization of the databases themselves?

\section{Editor's note}

Due to space limitation, credits for the cover image of the May 2002 issue were omitted. They are included as follows: $\beta_{2} \mathrm{~m}$ structure (foreground) courtesy of $C$. Trinh; $\beta_{2} \mathrm{~m}$ fibril micrograph (background) courtesy of K. Hasegawa and H. Naiki. We apologize for any inconvenience this may have caused. 